\title{
Validation of a standardized mapping system of the hip joint for radial MRA sequencing
}

\author{
Frank M. Klenke • Daniel B. Hoffmann • Brian J. Cross • \\ Klaus A. Siebenrock
}

Received: 7 April 2014 /Revised: 19 September 2014 / Accepted: 28 September 2014 / Published online: 14 October 2014

(C) ISS 2014

\begin{abstract}
Objective Intraarticular gadolinium-enhanced magnetic resonance arthrography (MRA) is commonly applied to characterize morphological disorders of the hip. However, the reproducibility of retrieving anatomic landmarks on MRA scans and their correlation with intraarticular pathologies is unknown. A precise mapping system for the exact localization of hip pathomorphologies with radial MRA sequences is lacking. Therefore, the purpose of the study was the establishment and validation of a reproducible mapping system for radial sequences of hip MRA.

Materials and methods Sixty-nine consecutive intraarticular gadolinium-enhanced hip MRAs were evaluated. Radial sequencing consisted of 14 cuts orientated along the axis of the femoral neck. Three orthopedic surgeons read the radial sequences independently. Each MRI was read twice with a minimum interval of 7 days from the first reading. The intraand inter-observer reliability of the mapping procedure was determined.

Results A clockwise system for hip MRA was established. The teardrop figure served to determine the 6 o'clock position of the acetabulum; the center of the greater trochanter served to determine the 12 o'clock position of the femoral head-neck junction. The intra- and inter-observer ICCs to retrieve the correct 6/12 o'clock positions were $0.906-0.996$ and 0.978 0.988 , respectively.

Conclusions The established mapping system for radial sequences of hip joint MRA is reproducible and easy to perform.
\end{abstract}

Keywords Hip $\cdot$ Magnetic resonance arthrography $\cdot$ Radial sequencing $\cdot$ Anatomic mapping

F. M. Klenke $(\bowtie) \cdot$ D. B. Hoffmann · B. J. Cross $\cdot$ K. A. Siebenrock Department of Orthopedic Surgery, Inselspital, Bern University Hospital, 3010 Bern, Switzerland

e-mail: frank.klenke@insel.ch

\section{Introduction}

MRA is commonly employed to localize and characterize intraarticular pathomorphologies of the hip and their secondary lesions. In this regard, MRA has been shown to be the most reliable imaging technique for disorders of the hip available today. In previous studies, the sensitivity and specificity of MRA in detecting lesions of the labrum and the cartilage ranged from $70 \%$ to $90 \%$ and $71 \%$ to $84 \%$, respectively [1-4]. The accuracy of coronal, sagittal, and radial MRA imaging in localizing intraarticular lesions was shown to be $69-91 \%[1,2,4]$. However, the reproducibility of identifying the exact anatomic location of a specific intraarticular pathology with MRA is unknown. Exact localization of morphologic changes influences the decision-making of whether to choose surgical or non-surgical treatment and determines the appropriate surgical strategy. Radial MRA sequences seem to be well suited to localize hip pathomorphologies reliably. They should avoid positional errors introduced by a varying pelvic tilt or different rotational positions of the femur during image acquisition. However, a precise mapping system for the localization of hip pathomorphologies with radial MRA sequences is lacking. Therefore, the purpose of the study was the establishment and validation of a reproducible mapping system for radial sequences of hip MRA based on anatomic landmarks.

\section{Materials and methods}

Magnetic resonance arthrography of 69 hips in 66 individuals (29 male, 37 female) with pathomorphologies of the hip joint were retrospectively reviewed. Pathomorphologies included femoroacetabular impingement (FAI, $n=39$ ), developmental dysplasia of the hip (DDH, $n=14$ ), Legg-Calvé-Perthes disease (LCP, $n=5$ ), slipped capital femoral epiphysis (SCFE, $n=$ 
4), acetabular retroversion $(n=3)$, and posttraumatic alterations $(n=4)$. Fifty-seven MRA were performed on nonoperated hips. Twelve MRA were performed postoperatively. Surgeries included surgical hip dislocations $(n=5)$, surgical hip dislocation with relative femoral neck lengthening $(n=1)$, periacetabular osteotomies $(n=2)$, intertrochanteric osteotomies $(n=2)$, prophylactic pinning of the femoral head $(n=1)$, and open reduction and internal fixation of a femoral neck fracture $(n=1)$. The mean patient age at the time of MRA was 31.3 years (range, 11-69 years). Institutional review board approval was obtained for this study.

MRA was performed on a 1.5-T high-field scanner (Siemens, Erlangen, Germany) by use of a flexible surface body coil for high spatial resolution. Intraarticular contrast enhancement was achieved with injection of $10-20 \mathrm{ml}$ of saline solution - diluted gadolinium diethylene triamine pentaacetic acid (DTPA) (Dotarem, 1:200; Guebert, Paris, France) under fluoroscopic guidance. In addition to the standard MRI protocol, radial proton density-weighted sequences (TR 2000, TE 15, field of view: $260 \times 260-\mathrm{mm}, 266 \times 512$ matrix, 14 slices) were oriented along the axis of the femoral neck (Fig. 1a). The images were performed based on a sagittal oblique localizer (Fig. 1b), marked on the proton densityweighted coronal sequence. Patients were placed in a supine position with the leg in $0^{\circ}$ (neutral) rotation. Thus, the relative orientation of entry level the acetabulum to the longitudinal axial of the femoral neck differed by the natural antetorsion of the proximal femur.

The validation of the radial sections was performed by three orthopedic surgeons trained on the radiographic evaluation of pathomorphologies of the hip joint. Each observer performed two readings on each radial imaging; the minimum interval between the readings was 7 days. At the femur, the most prominent contour of the greater trochanter served to define the 12 o'clock (superior) and 6 o'clock (inferior) position of the femoral head-neck junction (Fig. 1c). At the acetabulum, the six o'clock (inferior) position was defined by the deepest section of the fossa acetabuli (teardrop figure, Fig. 1d). Based on the determination of the 6 o'clock position, the opposite side of the acetabulum defined the 12 o'clock (superior) position. Starting from the 12 o'clock position the radial slices were translated into an hourly orientation. As 14 slices were acquired for each radial imaging, each slice corresponded to $12.85^{\circ}$ or 25.7 angular minutes. The
Fig. 1 a Schematic drawing depicting the orientation of the radial sections along the axis of the femoral neck. b MRA sagittal oblique localizer showing the orientation of the radial sections through the femoral neck and the acetabulum. c, d Radial MRA sections showing the 6/12 0'clock positions of the femoral head neck junction (c contour of the greater trochanter marked with arrowheads) and the acetabulum (d teardrop figure marked with asterisk) of the same hip. Note that the femoral 6/12 o'clock position (section no. 6) is not represented by the same section as the acetabular 6/12 o'clock position (section no. 9)
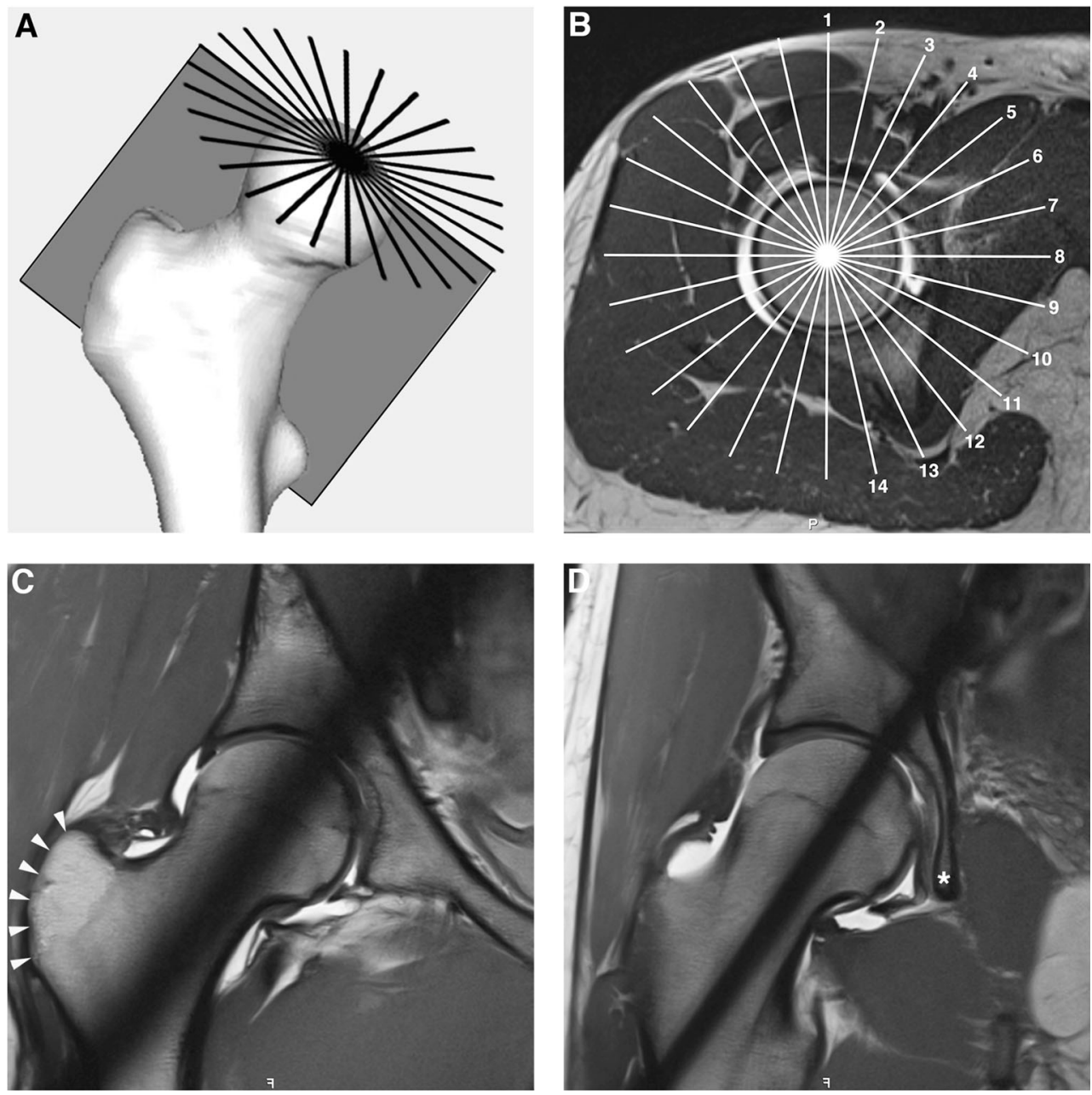
following protocol was used to translate the slicing of the radial images into an approximate hourly orientation.

- 6/12 o'clock: slice 1

- 1/7 o'clock: slice 3

- 2/8 o'clock: slice 6

- 3/9 o'clock: slice 8

- 4/10 o'clock: slice 10

- 5/11 o'clock: slice 13

The 3 o'clock position was defined as anterior and the 9 o'clock position as posterior for the femur and the acetabulum. To this end, all evaluations were converted to right hips.

The intraclass correlation coefficient (ICC) was used to calculate the level of agreement among two or more continuous variables [5]. According to Landis and Koch, the kappa coefficient served as the parameter of the strength of the agreement: $0=$ poor, $0.01-0.20=$ slight, $0.21-0.40=$ fair, $0.41-0.60=$ moderate, $0.61-0.80=$ substantial, and $0.81-1.0=$ almost perfect [5]. Cronbach's alpha coefficients were calculated to determine internal consistency. Inter- and intra-observer ICCs were calculated for the agreement of the determination of the $6 / 12$ o'clock and $3 / 9$ o'clock positions of the femoral head and the acetabulum. SPSS statistics was used for data analysis (IBM SPSS Statistics, Version 19 for Mac OSX).

\section{Results}

As shown in Table 1, the strength of the agreements for the intra-observer ICC between two consecutive measurements was "almost perfect" for the reliability to identify the $6 / 12$ o'clock positions of the femur as well as the acetabulum (0.906-0.996). There were no differences in the intraobserver ICC found between the three observers $(p=0.938)$. The intraobserver ICC was identical for the determination of the 6/12 o'clock positions and the 3/9 o'clock positions (data not shown). The interobserver ICC for the retrieval of the 6/12 o'clock positions was $0.978-0.988$ at the femur and $0.838-0.889$ at the acetabulum. No significant differences between the ICCs at the femur and the acetabulum were found $(p=0.881)$.

The localization of the 6/12 o'clock positions at the acetabulum and the femur differed by a means of 2.7 slices (range, 1-5 slices). In none of the three observers an exact agreement of the slice numbers determining the acetabular and the femoral 6/12 o'clock positions was found. At the acetabulum, the slice numbers determining the $6 / 12$ o'clock positions ranged from 6 to 10 . On the femoral side, a range of slice numbers from 5 to 12 represented the $6 / 12$ o'clock positions.

Subgroup analysis showed that there were no differences in intra- and inter-observer reliability between hips with femoroacetabular impingement and those with more severe alterations of the morphology of the hip joint (DDH, LCP, SCFE, acetabular retroversion; Tables 2 and 3). There were no differences in intra-observer reliability found independent of whether previous surgery of the respective hip had been performed. Analysis of interobserver ICCs revealed a significantly lower reliability for the correct determination of the $6 / 12$ o'clock positions of the acetabulum in operated hips as compared to non-operated hips $(p=0.045)$. In hips that had undergone previous surgery, interobserver ICCs were "substantial" (0.761-0.809) while they were "almost perfect" $(0.848-0.881)$ in native hips. The interobserver reliability of the determination of the femoral 6/12 o'clock positions was not influenced by previous surgeries (Tables 2 and 3).

\section{Discussion}

Reliable and exact spatial determination of hip joint pathologies is becoming increasingly important. The identification of pathologies including cartilage damage, lesions of the labrum, decreased head-neck offset, and acetabular retroversion delivers important information on the patients' individual prognosis [6-12]. Exact localization and characterization of morphologic changes is essential to determine the appropriate treatment strategy of hip joint pathomorphologies.

Radial sequencing of the hip joint achieves $360^{\circ}$ imaging of the acetabulum and the femoral head and neck. Radial sequences are well established to describe pathomorphologies
Table 1 Intraclass correlation coefficients for the determination of the 6/12 o'clock positions in 69 hips

\begin{tabular}{|c|c|c|c|c|c|c|c|}
\hline & & \multicolumn{2}{|c|}{ Observer I } & \multicolumn{2}{|c|}{ Observer II } & \multicolumn{2}{|c|}{ Observer III } \\
\hline & & Femur & Acetabulum & Femur & Acetabulum & Femur & Acetabulum \\
\hline \multirow[t]{2}{*}{ Observer I } & Femur & 0.991 & - & 0.978 & - & 0.988 & - \\
\hline & Acetabulum & - & 0.939 & - & 0.838 & - & 0.889 \\
\hline \multirow[t]{2}{*}{ Observer II } & Femur & 0.978 & & 0.992 & - & 0.982 & - \\
\hline & Acetabulum & - & 0.838 & - & 0.919 & - & 0.880 \\
\hline \multirow[t]{2}{*}{ Observer III } & Femur & 0.988 & & 0.982 & - & 0.996 & - \\
\hline & Acetabulum & - & 0.889 & - & 0.880 & - & 0.906 \\
\hline
\end{tabular}


Table 2 Mean intraobserver ICCs for the determination of the 6/12 o'clock positions in different pathomorphologies of the hip as well as in native and surgically treated hips

\begin{tabular}{|c|c|c|c|c|c|c|c|c|}
\hline & \multicolumn{4}{|c|}{ Intraobserver ICC } & \multicolumn{4}{|c|}{ Interobserver ICC } \\
\hline & \multicolumn{2}{|l|}{ FAI } & \multicolumn{2}{|c|}{ DDH/LCP/SCFE/RETRO } & \multicolumn{2}{|l|}{ FAI } & \multicolumn{2}{|c|}{ DDH/LCP/SCFE/RETRO } \\
\hline & Femur & Acetabulum & Femur & Acetabulum & Femur & Acetabulum & Femur & Acetabulum \\
\hline Observer I vs. Observer II & 0.990 & 0.951 & 0.989 & 0.929 & 0.973 & 0.844 & 0.981 & 0.867 \\
\hline Observer I vs. Observer III & 0.992 & 0.904 & 0.993 & 0.931 & 0.983 & 0.870 & 0.988 & 0.913 \\
\hline Observer II vs. Observer III & 0.994 & 0.886 & 0.996 & 0.936 & 0.978 & 0.817 & 0.984 & 0.892 \\
\hline
\end{tabular}

of the hip joint [3, 13-16]. In order to achieve accurate and precise localization of intraarticular pathologies, radial sequences need to be evaluated according to a standardized system. However, such mapping system is lacking. Furthermore, the inter- and intra-observer reliabilities of the localization of femoral and acetabular landmarks with radial sequence MRA are not well known.

This study has several limitations due to its design as a pure imaging study without evaluation of intraoperative findings. The localization of the anatomic landmarks, namely the teardrop figure and the superolateral acetabular wall as well as the center of the greater trochanter, were not correlated with the intraoperative localization of these landmarks. Furthermore, the accuracy to predict intraarticular pathologies such as labral tears and cartilage lesions was not analyzed. This would have required intraoperative information of all patients. However, only 33 of the 69 hips have undergone surgery following their MRI. This study was designed to establish a standardized mapping system for radial sequences and to provide a proof of principle for a reliable and reproducible definition of intraarticular landmarks. Future studies will need to analyze the correlation of the imaging evaluation with the intraoperative determination of anatomic landmarks and intraarticular pathologies.

This study shows that radial MRA sequencing of the hip joint can be used to map the acetabulum as well as the femoral head-neck junction reliably. The mapping system can be translated into an easy-to-read hourly orientation. Orientation of the 6/12 o'clock positions on the basis of precisely retrievable anatomic landmarks resulted in "almost perfect" inter- and intra-observer ICCs according to Landis and Koch [5]. Furthermore, the maximum deviation in determining the 6/12 o'clock positions was one MRI cut corresponding to a maximum error of precision of 25.7 angular minutes.

Hourly mapping of radial MRA sections proved to have a high inter- and intra-observer reliability independent of the underlying pathomorphology. Variations of the orientation of the acetabulum in DDH and acetabular retroversion as well as deformities of the femoral head-neck junction and the greater trochanter in SCFE and LCP disease, respectively, did not influence the reliability of the mapping method. Previous surgical interventions altering the anatomy of the acetabulum and/or the proximal femur and eliciting metal artifacts did not affect femoral mapping. However, surgical interventions of the hip decreased the interobserver reliability of the acetabular mapping significantly without influencing the intraobserver reliability. Still, interobserver ICCs for acetabular mappings in hips that had undergone surgery were "substantial".

Blankenbaker et al. [17] have previously described a clockwise MRA-based reading system to localize labral tears. Comparison of hip MRA with arthroscopic findings showed that the difference of the localization of the labral tears was lesser or equal $1 \mathrm{~h}$ in $86 \%$ of the hips. Thus, the mapping system provided good accuracy in localizing pathomorphologies of the acetabular labrum. However, it has not been validated for the localization of pathomorphologies of the proximal femur. Furthermore, the mapping system required coronal, oblique

Table 3 Mean interobserver ICCs for the determination of the 6/12 o'clock positions in different pathomorphologies of the hip as well as in native and surgically treated hips

\begin{tabular}{|c|c|c|c|c|c|c|c|c|}
\hline & \multicolumn{4}{|c|}{ Intraobserver ICC } & \multicolumn{4}{|c|}{ Interobserver ICC } \\
\hline & \multicolumn{2}{|c|}{ No prev. surgery } & \multicolumn{2}{|c|}{ Prev. surgery } & \multicolumn{2}{|c|}{ No prev. surgery } & \multicolumn{2}{|c|}{ Prev. surgery } \\
\hline & Femur & Acetabulum & Femur & Acetabulum & Femur & Acetabulum & Femur & Acetabulum \\
\hline Observer I vs. Observer II & 0.990 & 0.927 & 0.991 & 0.969 & 0.977 & 0.848 & 0.977 & $0.761^{*}$ \\
\hline Observer I vs. Observer III & 0.987 & 0.904 & 0.996 & 0.951 & 0.988 & 0.879 & 0.985 & $0.809^{*}$ \\
\hline Observer II vs. Observer III & 0.994 & 0.912 & 1.000 & 0.849 & 0.980 & 0.881 & 0.983 & $0.781^{*}$ \\
\hline
\end{tabular}


axial, and sagittal imaging planes to achieve good accuracy. The presented system requires only a single radial MRA sequence to achieve accurate mapping of the acetabulum and the proximal femur. Radial sequences are oriented perpendicular to the acetabular rim and along the axis of the femoral neck, respectively. Thus, the accuracy of a mapping system based on radial images is less prone to artifacts introduced by variations of the pelvic tilt or the rotation of the femur. However, the 6/12 o'clock positions of the acetabulum and the femur are not represented by the same slice of a radial MRI. Therefore, the anatomic localization has to be carried out separately for the acetabulum and the femur. In clinical practice, the femoral 12 o'clock position is often used for the localization of morphologic changes of the acetabulum. This approach will result in incorrect localizations of morphologic changes at the acetabular side. Such misinterpretations may mislead the physician in his/her decision-making on how to address the respective pathomorphology. Furthermore, inconsistencies in the localization of morphologic changes compromise the interpretation of the radiologist's report and the communication between the radiologist and the surgeon.

Differences in the determination of the $6 / 12$ o'clock and 3/9 o'clock positions were not observed in the present study. Nevertheless, radial sequencing using a grid with 14 slices may be prone to false readings due to the irregular translation of the slices into an hourly orientation. Therefore, radial sequencing with 12 slices may be easier to apply in clinical practice. Our MRA protocol will be adapted accordingly. The slightly decreased spatial resolution associated with a reduced number of slices will not decrease the precision of radial MRA imaging.

A reproducible, easy-to-perform mapping system for radial sequences of hip joint MRA based on anatomic landmarks has been established and validated. This method proved to have a very high inter- and intra-observer reliability. This system will help to localize morphologic changes of the hip joint precisely thereby achieving more consistent agreements of preoperative MRA imaging and intra-operative findings. Furthermore, an accurate identification of morphologic changes will facilitate longitudinal long-term studies. Further studies will be necessary to correlate the presented MRA-based mapping system with the intraoperative localization of anatomic landmarks and intraarticular pathologies.

Conflict of interest All authors declare that they have no conflicts of interest in connection with the submitted article.

\section{References}

1. Czerny C, Hofmann S, Neuhold A, Tschauner C, Engel A, Recht MP, et al. Lesions of the acetabular labrum: accuracy of MR imaging and MR arthrography in detection and staging. Radiology. 1996;200: 225-30.

2. Czerny C, Hofmann S, Urban M, Tschauner C, Neuhold A, Pretterklieber M, et al. MR arthrography of the adult acetabular capsular-labral complex: correlation with surgery and anatomy. AJR Am J Roentgenol. 1999;173:345-9.

3. Dudda M, Mamisch TC, Krueger A, Werlen S, Siebenrock KA, Beck M. Hip arthroscopy after surgical hip dislocation: is predictive imaging possible? Arthrosc J Arthrosc Relat Surg Off Publ Arthrosc Assoc N Am Int Arthrosc Assoc. 2011;27:486-92.

4. Schmid MR, Notzli HP, Zanetti M, Wyss TF, Hodler J. Cartilage lesions in the hip: diagnostic effectiveness of MR arthrography. Radiology. 2003;226:382-6.

5. Landis JR, Koch GG. The measurement of observer agreement for categorical data. Biometrics. 1977;33:159-74.

6. Berton C, Bocquet D, Krantz N, Cotten A, Migaud H, Girard J. Shelf arthroplasties long-term outcome: influence of labral tears. A prospective study at a minimal 16 years' follow up. Orthop Traumatol Surg Res. 2010;96:753-9.

7. Byrd JW, Jones KS. Prospective analysis of hip arthroscopy with 2year follow-up. Arthrosc J Arthrosc Relat Surg Off Publ Arthrosc Assoc N Am Int Arthrosc Assoc. 2000;16:578-87.

8. Ganz R, Parvizi J, Beck M, Leunig M, Notzli H, Siebenrock KA. Femoroacetabular impingement: a cause for osteoarthritis of the hip. Clin Orthop Relat Res. 2003;417:112-20.

9. Gdalevitch M, Smith K, Tanzer M. Delamination cysts: a predictor of acetabular cartilage delamination in hips with a labral tear. Clin Orthop Relat Res. 2009;467:985-91.

10. Kim WY, Hutchinson CE, Andrew JG, Allen PD. The relationship between acetabular retroversion and osteoarthritis of the hip. J Bone Joint Surg Br Vol. 2006;88:727-9.

11. Lequesne M, Bellaiche L. Anterior femoroacetabular impingement: an update. Joint Bone Spine Rev Rhum. 2012;79:249-55.

12. O'Leary JA, Berend K, Vail TP. The relationship between diagnosis and outcome in arthroscopy of the hip. Arthrosc J Arthrosc Relat Surg Off Publ Arthrosc Assoc N Am Int Arthrosc Assoc. 2001;17: $181-8$.

13. Dudda M, Albers C, Mamisch TC, Werlen S, Beck M. Do normal radiographs exclude asphericity of the femoral head-neck junction? Clin Orthop Relat Res. 2009;467:651-9.

14. Plotz GM, Brossmann J, von Knoch M, Muhle C, Heller M, Hassenpflug J. Magnetic resonance arthrography of the acetabular labrum: value of radial reconstructions. Arch Orthop Trauma Surg. 2001;121:450-7.

15. Rakhra KS, Sheikh AM, Allen D, Beaule PE. Comparison of MRI alpha angle measurement planes in femoroacetabular impingement. Clin Orthop Relat Res. 2009;467:660-5.

16. Yoon LS, Palmer WE, Kassarjian A. Evaluation of radial-sequence imaging in detecting acetabular labral tears at hip MR arthrography. Skeletal Radiol. 2007;36:1029-33.

17. Blankenbaker DG, De Smet AA, Keene JS, Fine JP. Classification and localization of acetabular labral tears. Skeletal Radiol. 2007;36: 391-7. 